\title{
Simple Synthesis and Enhanced Performance of Graphene Oxide-Gold Composites
}

\author{
Min Song, Lulu Yu, and Yimin Wu \\ Key Laboratory of Energy Thermal Conversion and Control, School of Energy and Environment, Southeast University, \\ Ministry of Education, Nanjing 210096, China \\ Correspondence should be addressed to Min Song, sm@seu.edu.cn
}

Received 23 January 2012; Accepted 10 March 2012

Academic Editor: Anukorn Phuruangrat

Copyright ( $(2012$ Min Song et al. This is an open access article distributed under the Creative Commons Attribution License, which permits unrestricted use, distribution, and reproduction in any medium, provided the original work is properly cited.

Graphene oxide-gold composites were prepared by one-step reaction in aqueous solution, where the gold nanoparticles were deposited on the graphene oxide during their synthesis process. Transmission electron morphology, X-ray diffraction, Roman spectra, and UV-Vis absorption spectra were used to characterize the obtained composites. Furthermore, based on the BET analysis results, it was found that the surface area of the composite film was obviously enhanced compared with the synthesized graphene oxide. Electrochemical measurements indicated that the modification of the composites on electrode could efficiently enhance the voltammetric response, suggesting the potential application for making electrochemical sensors.

\section{Introduction}

Graphene, a two-dimensional material, has attracted tremendous attention from both the experimental and theoretical scientific communities due to its unique nanostructure and properties, such as the high thermal conductivity, high strength, and high specific surface area. These properties make graphene very promising for many applications such as solar cells, hydrogen storage [1], sensors [2], batteries [3], supercapacitors [4], and nanocomposites $[5,6]$.

Beside pure graphene, the study of grapheme-based nanocomposites is also important. The combination of graphene with inorganic particles, such as metallic, semiconducting, and insulating nanoparticles (NPs), may result in some excellent materials used for catalysts and batteries [7-10]. Graphene-based composite materials prepared by dispersing in polymers show significant improvement in the electronic and thermal conductivity $[11,12]$. Also, the integration of graphene with certain functional particles presents special features in the new hybrids that can be used in optical, electrical, catalysis, sensors, and so forth [13].

The graphene can be obtained in bulk quantity by chemical reduction of graphene oxide in solution [14-16].
However, the strong van der Waals interactions among these reduced graphene sheets result in their tendency to aggregate in solution. Both electrostatic stabilization [17] and chemical functionalization $[18,19]$ methods have been proven to be useful to suppress aggregation of exfoliated graphene sheets, such as the attachment of some molecules or polymers onto the sheets $[11,20]$. Furthermore, several investigations have been carried out to produce graphene-metal composites to prevent the restacking of these carbon sheets during the chemical reduction process $[8-10,21,22]$. Electrochemical deposition, metal evaporation, and hydrogen reduction of metallic salts-graphite composite can be used to prepare graphite-metal composites.

In this study, we have explored a new strategy to deposit gold nanoparticles on graphene oxide by one-step reaction in aqueous solution, where the gold nanoparticles are deposited on the graphene oxide during their synthesis process. The as synthesized composites are characterized by using transmission electron morphology, X-ray diffraction, Roman spectra, and UV-Vis absorption. Moreover, we investigate the signal enhancement of the composite by electrochemical detection, suggesting the potential application for electrochemical sensors. 


\section{Experimental Section}

2.1. Preparation of Graphene Oxide-Gold Composites. The natural graphite was bought from Sigma. The graphite oxide (GO) was prepared from purified natural graphite according to Hummers method. The $2 \mathrm{mg}$ GO was suspended in $1 \mathrm{wt} \%$ $\mathrm{HAuCl}_{4}$ solution $(2 \mathrm{~mL})$ by sonication for 5 minutes to make GO dispersed equably. The suspended solution was then diluted to $200 \mathrm{~mL}$ with doubly distilled water and heated to boiling while stirring. Afterward, $20 \mathrm{~mL}$ sodium citrates was added to the boiling solution which was kept heating for 30 min with magnetic stirring until the color of the solutions did not change. Then, the sample was cooled to room temperature, then separated in the centrifuge, and washed with doubly distilled water five times. The resulting products were dried in a vacuum oven at $80^{\circ} \mathrm{C}$ for $12 \mathrm{~h}$.

2.2. Characterization. TEM images were obtained by using JEM2000FX (JEOL). The composition and structure were analyzed using powder X-ray diffraction (XRD, Rigaku D/ max-2500 diffractometer with $\mathrm{Cu} \mathrm{K} \alpha$ radiation). BrunauerEmmett-Teller (BET) surface areas and pore volumes were measured on a Micromeritics ASAP 2020 sorptometer using nitrogen adsorption at $77 \mathrm{~K}$. UV-Vis absorption spectroscopy was performed on Hitachi U-4100. Raman spectra were recorded with surface enhanced Raman spectrophotometer (JY HR800).

2.3. Electrochemical Measurements. Initially, $1 \mathrm{mg}$ graphene oxide or graphene oxide-gold composites were suspend in $10 \mathrm{~mL}$ double-distilled water. Then $5 \mathrm{mLs}$ of the relative solution was spread evenly onto the surfaces of the GCE electrode. Afterwards, the electrode surfaces were completely dried under a flow of nitrogen.

Electrochemical measurements were performed on a $\mathrm{CHI}$ 660B electrochemical workstation at room temperature $\left(22 \pm 2{ }^{\circ} \mathrm{C}\right)$ under the nitrogen atmosphere. A three-electrode system was used in the relative electrochemical study, which contained the composites-modified glassy carbon electrode (GCE) as the working electrode, a Pt electrode as the counter electrode, and a saturated calomel electrode (SCE) as the reference electrode.

\section{Results and Discussion}

To demonstrate the effective coating of gold nanoparticles on the graphene oxide surface, TEM, XRD, Roman spectra, and UV-vis absorption spectra are used to characterize the graphene oxide and graphene oxide-Au composites. Figures 1(a) and 1(b) show the typical TEM images of the synthesized graphene oxide and graphene oxide-Au composites. The TEM image of graphene oxide nanosheets illustrates the flake-like shapes of graphene oxide. Transmission electron microscopy (TEM) micrographs demonstrate that the gold nanoparticles are deposited on graphene oxide sheets. In this image, $\mathrm{Au}$ nanoparticles appear as dark dots with a diameter of 20 to $30 \mathrm{~nm}$ on a lighter shaded substrate corresponding to the planar graphene oxide sheet.
Furthermore, Figure 1(c) shows the XRD patterns of the as-synthesized graphene oxide and Au-graphene oxide composites. From the results, we observe that GO shows position peak at $11.3^{\circ}$, suggesting the successful oxidation of the starting graphite. Meanwhile, the diffraction peaks of the Au-graphene oxide composite are similar to that of pure Au. The peaks at $2 \theta=39.9,46.2,67.9$, and $81.4^{\circ}$ can be assigned to the (111), (200), (220), and (311) crystalline planes of Au. In addition, we also observe that the regular layered structure of graphite oxide or graphite is destroyed. The possible mechanism is that the attached particles prevent the restacking of these carbon sheets and therefore the characteristic diffractions peaks of the layered structure disappear [23].

Figure 1(d) shows the Raman spectra of graphene oxide and graphene oxide-AuNPs composites. As for graphene oxide, the peaks at $1590 \mathrm{~cm}^{-1}$ (G band) and $1360 \mathrm{~cm}^{-1}$ (D band) are clearly present. In the meanwhile, the D-band and G-band for graphene oxide-AuNPs composites are also located at 1360 and $1590 \mathrm{~cm}^{-1}$, respectively. The ratio between the $\mathrm{D}$ and $\mathrm{G}$ bands $\left(I_{\mathrm{D}} / I_{\mathrm{G}}\right)$ can be used to estimate the degree of structural disorder. The Raman spectrum of graphene oxide-AuNPs composites material is very similar to the Raman spectrum of the graphene oxide with practically identical $\mathrm{D} / \mathrm{G}$ band ratio, indicating the nondestructive character of the method. However, the peak intensities of all relative bands for graphene oxide-AuNPs composites are obviously higher than those for the corresponding bands observed for the graphene oxide, suggesting a strong coating of the ligand on the graphene oxide surface. Furthermore, all Raman spectra have the same pattern, which implies that the binding procedure does not affect the structure of graphene.

The successful synthesis of AuNPs-decorated graphene oxide is confirmed by UV-vis spectroscopy (Figure 1(e)). The UV-vis spectrum of graphene oxide (curve a in Figure 1(e)) in water shows that no absorption peak from 300 to $700 \mathrm{~nm}$. As shown in Figure 1(e) (curve c), when AuNPs are decorated onto the graphene oxide surface, the absorption peaks of graphene oxide-AuNPs composites are observed at $520 \mathrm{~nm}$, which is corresponding to the absorption of AuNPs (curve b), indicating the formation of AuNPs.

The redox probe $\mathrm{Fe}(\mathrm{CN})_{6}{ }^{3-}$, which is sensitive to surface chemistry of carbon-based electrodes, was used to characterize the bare and modified GCEs. Figure 2 shows the enhanced electrochemical response of $\mathrm{K}_{3}(\mathrm{FeCN})_{6}$ at the respective modified glassy carbon electrodes. It is observed that as for the bare GCE, the peak current of $\mathrm{K}_{3}(\mathrm{FeCN})_{6}$ is $4.0 \times$ $10^{-5} \mathrm{~A}$, while the relevant peak current increases to $4.8 \times$ $10^{-5} \mathrm{~A}$ at pure graphene oxide modified GCE. Thus, the peak current intensity of $\mathrm{K}_{3}(\mathrm{FeCN})_{6}$ is increased about $20 \%$ in the presence of graphene oxide. In comparison, in the presence of graphene oxide-AuNPs composites, the respective peak current of $\mathrm{K}_{3}(\mathrm{FeCN})_{6}$ increases to $9.0 \times 10^{-5} \mathrm{~A}$. It appears that the peak current of $\mathrm{K}_{3}(\mathrm{FeCN})_{6}$ increases about $125 \%$ in the presence of graphene oxide-AuNPs composites under identical experimental conditions. The results indicate that the current response of $\mathrm{K}_{3}(\mathrm{FeCN})_{6}$ at the graphene oxideAuNPs composites modified electrode is much stronger than that of graphene oxide modified GCE as well as the bare 


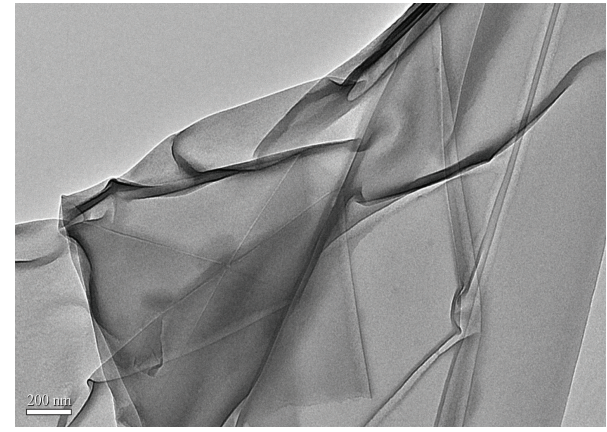

(a)

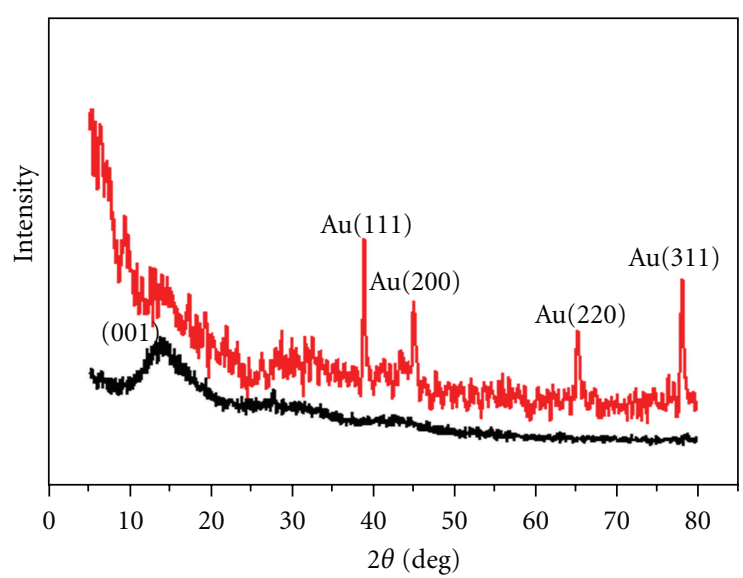

- a

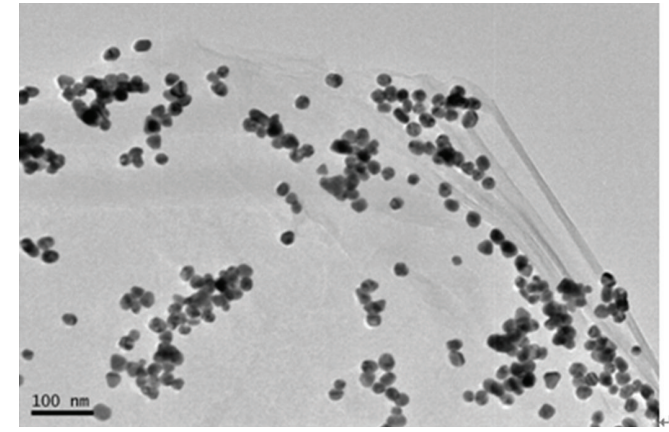

(b)

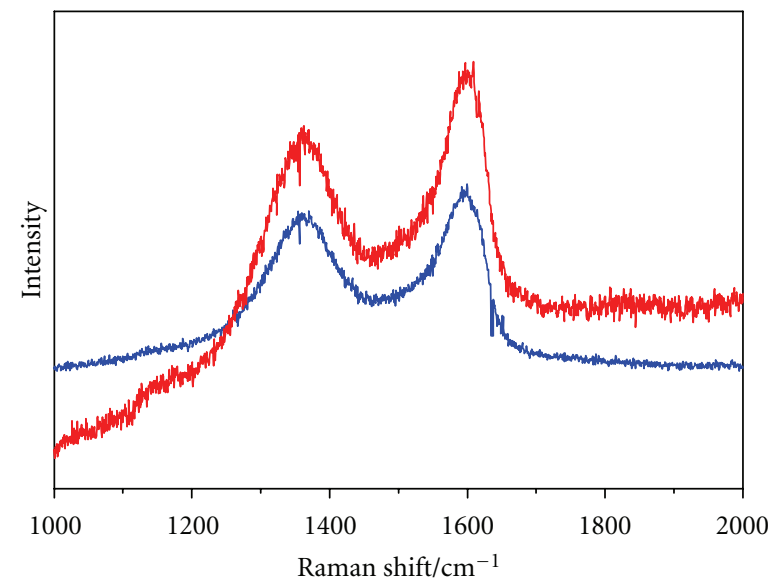

- a

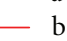

(d)

(c)

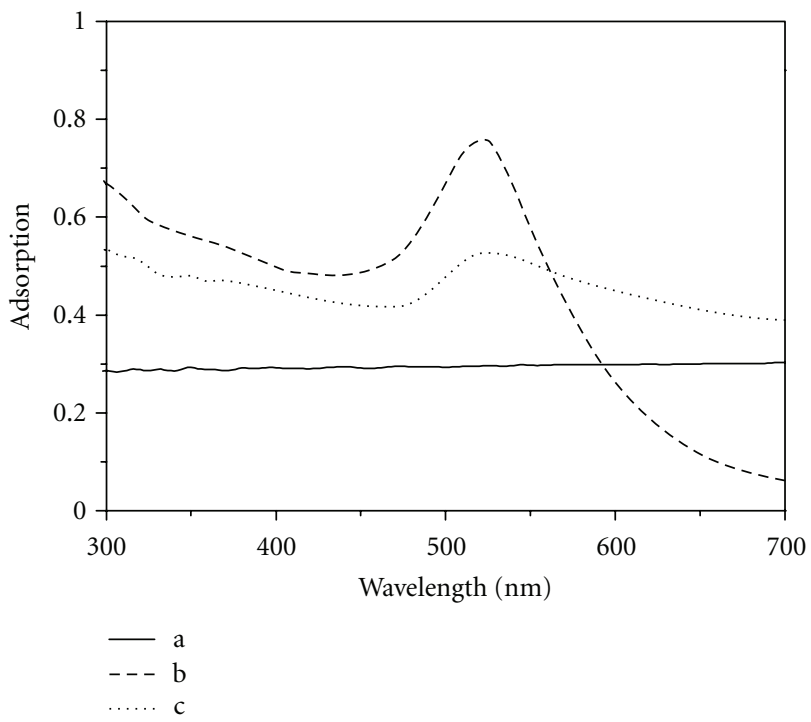

(e)

FIGURE 1: TEM images of the graphene oxide (a) and graphene oxide-Au composites (b). (c) XRD images of the graphite oxide (a) and graphene oxide-Au composites (b). (d) Raman spectra of the graphene oxide (a) and graphene oxide-Au composites (b). (e) UV-vis absorption spectra of the graphene oxide (a), Au nanoparticles (b) and graphene oxide-Au composites (c). 
TABLE 1: The texture property of graphene oxide and graphene oxide-AuNPs composites.

\begin{tabular}{lccc}
\hline Samples & & \multicolumn{2}{c}{ Properties } \\
& Pore diameter/nm & BET surface area/m $/ \mathrm{g}$ & Pore volume/cm $/ \mathrm{g}$ \\
\hline Graphene oxide & 31.5 & 23 & 0.18 \\
Graphene oxide -AuNPs composites & 4.18 & 985 & 1.03 \\
\hline
\end{tabular}

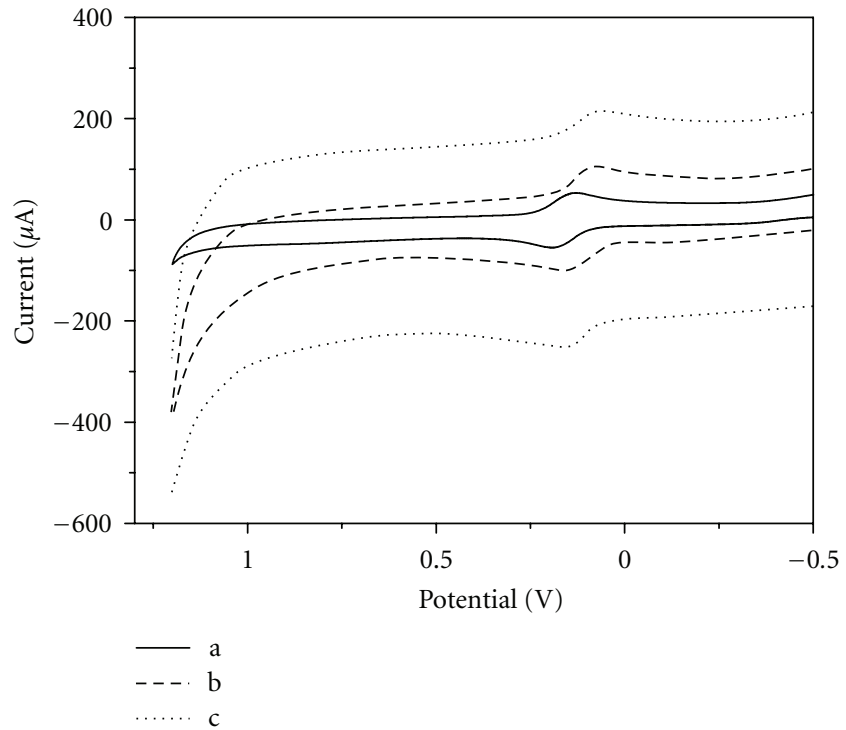

Figure 2: Cyclic voltammogram of $\mathrm{K}_{3}(\mathrm{FeCN})_{6}$ at the bare GCE (a), pure graphene oxide modified GCE (b), and graphene oxide-AuNPs composites modified GCE (c).

GCE. The enhanced current response on composites should be ascribed to the effective intercalation of Au NPs onto the graphene oxide sheets, which contribute to the surface area enhancement of the composite film. To confirm this, the surface area and pore structures properties of graphene oxide and graphene oxide-AuNPs composites are elucidated by BET methods in Table 1. From the results, we can obviously observed that the surface area of composites increased by incorporation of Au NPs.

\section{Conclusion}

In summary, gold nanoparticles are attached on the graphene oxide surface during their synthesis process. Our results indicate that the premixing of graphene oxide with $\mathrm{HAuCl}_{4}$ solution could contribute to the distribution of $\mathrm{HAuCl}_{4}$ molecule on the graphene oxide surface and further induce the nice deposition of gold nanoparticles on graphene oxide sheets. The enhancement of surface area and respective electrochemical response shows a potential application of the composite for electrochemical sensing.

\section{Acknowledgments}

The authors are grateful for the National Natural Science Foundation of China (20907008) and the financial support of the National Hi-tech Research and Development Program of China (973) (2010CB732206 and 2011CB201505), Specialized Research Fund for the Doctoral Program of Higher Education (20090092120010). Dr. M. Song specially thanks the support of the Foundation for Southeast University excellent young teacher.

\section{References}

[1] X. Wang, L. Zhi, and K. Müllen, "Transparent, conductive graphene electrodes for dye-sensitized solar cells," Nano Letters, vol. 8, no. 1, pp. 323-327, 2008.

[2] J. D. Fowler, M. J. Allen, V. C. Tung, Y. Yang, R. B. Kaner, and B. H. Weiller, "Practical chemical sensors from chemically derived graphene," ACS Nano, vol. 3, no. 2, pp. 301-306, 2009.

[3] C. Wang, D. Li, C. O. Too, and G. G. Wallace, "Electrochemical properties of graphene paper electrodes used in lithium batteries," Chemistry of Materials, vol. 21, no. 13, pp. 26042606, 2009.

[4] Y. Wang, Z. Shi, Y. Huang et al., "Supercapacitor devices based on graphene materials," Journal of Physical Chemistry C, vol. 113, no. 30, pp. 13103-13107, 2009.

[5] G. Eda and M. Chhowalla, "Graphene-based composite thin films for electronics," Nano Letters, vol. 9, no. 2, pp. 814-818, 2009.

[6] J. L. Vickery, A. J. Patil, and S. Mann, "Fabrication of graphene-polymer nanocomposites with higher-order three-dimensional architectures," Advanced Materials, vol. 21, no. 21, pp. 2180-2184, 2009.

[7] R. Patakfalvi, D. Diaz, P. Santiago-Jacinto, G. RodriguezGattorno, and R. Sato-Berru, "Anchoring of silver nanoparticles on graphite and isomorphous lattices," Journal of Physical Chemistry C, vol. 111, no. 14, pp. 5331-5336, 2007.

[8] M. Shirai, K. Igeta, and M. Arai, "Formation of platinum nanosheets between graphite layers," Chemical Communications, no. 7, pp. 623-624, 2000.

[9] S. K. Shaikhutdinov and F. J. C. Santos Aires, "Evolution of the rhodium colloid supported on graphite studied by atomic force microscopy in the tapping mode," Langmuir, vol. 14, no. 13, pp. 3501-3505, 1998.

[10] X. Yang, Y. Makita, Z. H. Liu, and K. Ooi, "Novel synthesis of layered graphite oxide-birnessite manganese oxide nanocomposite," Chemistry of Materials, vol. 15, no. 6, pp. 1228-1231, 2003.

[11] S. Stankovich, D. A. Dikin, G. H. B. Dommett et al., "Graphene-based composite materials," Nature, vol. 442, no. 7100, pp. 282-286, 2006.

[12] A. Yu, P. Ramesh, M. E. Itkis, E. Bekyarova, and R. C. Haddon, "Graphite nanoplatelet-epoxy composite thermal interface materials," Journal of Physical Chemistry C, vol. 111, no. 21, pp. 7565-7569, 2007.

[13] H. Yang, Q. Zhang, C. Shan, F. Li, D. Han, and L. Niu, "Stable, conductive supramolecular composite of graphene sheets with 
conjugated polyelectrolyte," Langmuir, vol. 26, no. 9, pp. 6708-6712, 2010.

[14] G. Eda, G. Fanchini, and M. Chhowalla, "Large-area ultrathin films of reduced graphene oxide as a transparent and flexible electronic material," Nature Nanotechnology, vol. 3, no. 5, pp. 270-274, 2008.

[15] M. J. McAllister, J. L. Li, D. H. Adamson et al., "Single sheet functionalized graphene by oxidation and thermal expansion of graphite," Chemistry of Materials, vol. 19, no. 18, pp. 43964404, 2007.

[16] G. Wang, J. Yang, J. Park et al., "Facile synthesis and characterization of graphene nanosheets," Journal of Physical Chemistry $C$, vol. 112, no. 22, pp. 8192-8195, 2008.

[17] D. Li, M. B. Müller, S. Gilje, R. B. Kaner, and G. G. Wallace, "Processable aqueous dispersions of graphene nanosheets," Nature Nanotechnology, vol. 3, no. 2, pp. 101-105, 2008.

[18] S. Niyogi, E. Bekyarova, M. E. Itkis, J. L. McWilliams, M. A. Hamon, and R. C. Haddon, "Solution properties of graphite and graphene," Journal of the American Chemical Society, vol. 128, no. 24, pp. 7720-7721, 2006.

[19] A. B. Bourlinos, D. Gournis, D. Petridis, T. Szabó, A. Szeri, and I. Dékány, "Graphite oxide: chemical reduction to graphite and surface modification with primary aliphatic amines and amino acids," Langmuir, vol. 19, no. 15, pp. 6050-6055, 2003.

[20] S. Stankovich, R. D. Piner, X. Chen, N. Wu, S. T. Nguyen, and R. S. Ruoff, "Stable aqueous dispersions of graphitic nanoplatelets via the reduction of exfoliated graphite oxide in the presence of poly(sodium 4-styrenesulfonate)," Journal of $\mathrm{Ma}$ terials Chemistry, vol. 16, no. 2, pp. 155-158, 2006.

[21] G. Zhu, L. Pan, H. Sun et al., "Electrophoretic Deposition of a reduced graphene-Au nanoparticle composite film as counter electrode for CdS quantum dot-sensitized solar cells," Chemphyschem, vol. 13, pp. 769-773, 2012.

[22] Y. Fan, H. Cheng, C. Zhou et al., "Honeycomb architecture of carbon quantum dots: a new efficient substrate to support gold for stronger SERS," Nanoscale, vol. 4, pp. 1776-1781, 2012.

[23] C. Xu, X. Wang, and J. Zhu, "Graphene-metal particle nanocomposites," Journal of Physical Chemistry C, vol. 112, no. 50, pp. 19841-19845, 2008. 

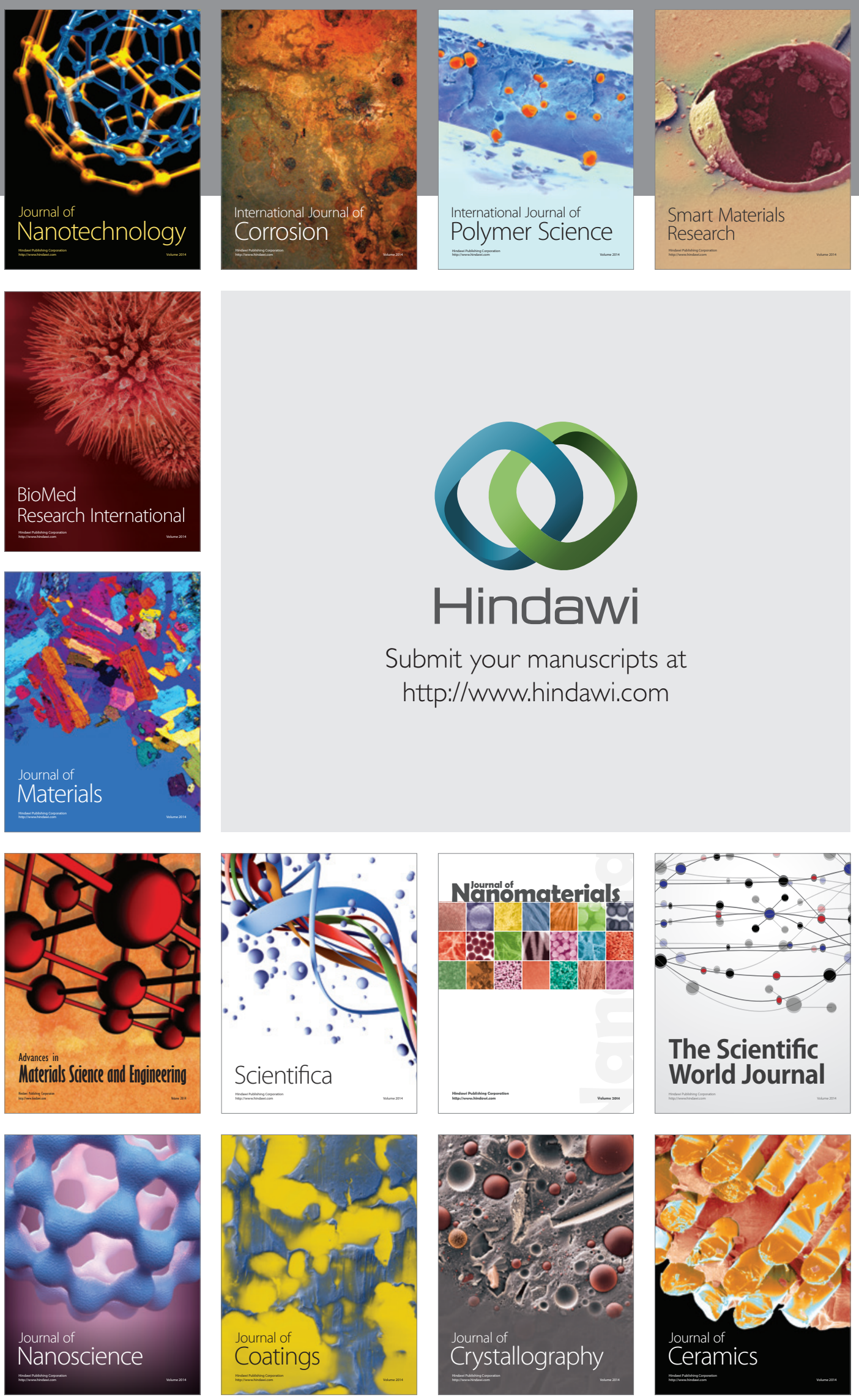

The Scientific World Journal

Submit your manuscripts at

http://www.hindawi.com

\section{World Journal}

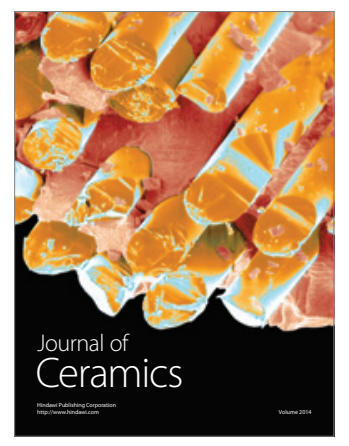

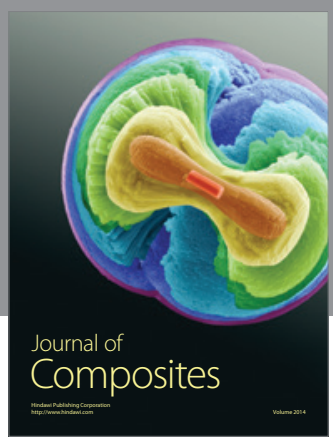
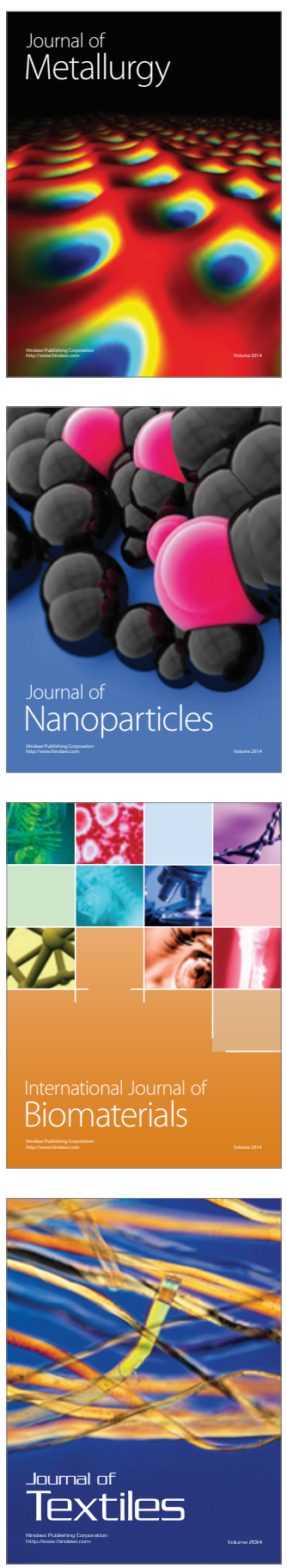